\title{
La responsabilidad ética, medioambiental y profesional como competencia transversal (CT07) en el Grado de Gestión y Administración Pública
}

\author{
Sosa-Espinosa, Asenet ${ }^{\mathrm{a}}$; Portillo-Poblador, Nuria ${ }^{\mathrm{b}}$ y González-de-Julián, Silvia ${ }^{\mathrm{c}}$ \\ aProfesora Titular E.U., Área de Sociología, Dpto. Urbanismo. Universitat Politècnica de València. España, \\ assoes@urb.upv.es, ${ }^{b}$ Profesora Colaboradora, Dpto. de Estadística e Investigación Operativa Aplicadas y Calidad. \\ Universitat Politècnica de València nportillo@eio.upv.es ${ }^{c}$ Profesora Asociada, Dpto. De Economía y Ciencias Sociales, \\ Universitat Politècnica de València, silgonde@upv.es
}

\begin{abstract}
This document gathers the results and reflections of the team work realized by the professors that impart subjects that are control points for the evaluation of the transversal competence 7: Ethical responsibility, environmental and professional, included in the Grade of Management and Public Administration (GGAP), in the Faculty of Business Administration of the Polytechnic University of Valencia (UPV). Among the results of this work, active learning highlights as a methodology, materialized in three types that have been applied: learn doing, reflective learning and service learning. Thereby, the experience and learning obtained by the teachers in terms of potentialities and limitations during the evaluation of this competence is synthesized.
\end{abstract}

Keywords: competencies, ethics, environment, professionalism, mainstreaming, learn doing, reflective learning, service learning, public management, public administration.

\section{Resumen}

Este documento recoge los resultados y reflexiones del trabajo de equipo realizado por el profesorado que imparte asignaturas que son punto de control para la evaluación de la Competencia Transversal 07: responsabilidad ética, medioambiental y profesional, dentro del Grado de Gestión y Administración Pública, en la Facultad de Administración y Dirección de Empresas de la Universitat Politècnica de València. Entre los resultados de dicho trabajo destaca el aprendizaje activo como vía metodológica, concretado en tres tipos que se han aplicado: aprender haciendo, aprendizaje reflexivo y aprendizaje servicio. Así también, se sintetiza la experiencia y aprendizaje obtenido por los y las docentes en términos de potencialidades y limitaciones en la evaluación de dicha competencia.

Palabras clave: competencias, ética, medioambiente, profesionalidad, transversalidad, aprender haciendo, aprendizaje reflexivo, aprendizaje servicio, gestión pública, administración pública. 


\section{Competencias y transversalidad}

Genéricamente, cuando hablamos de competencias, nos referimos a aptitudes, idoneidades o pericias a la hora de hacer algo o intervenir en un asunto determinado. Llevado al sistema educativo, y específicamente a la educación superior, a las instituciones universitarias y sus planes de estudios, hablamos de la obligación de especificar qué perfil tienen los egresados, es decir, las capacidades para las que habilita la titulación realizada (Gimeno, et.al., 2008: 11-12), relacionadas con el objetivo saber hacer que ya recogía el informe Delors (1996), pero también con saber aprehender. Esta obligación arranca con la declaración de Bolonia (1998) dentro del proceso de creación del Espacio Europeo de Educación Superior, como fórmula posible para homologar titulaciones universitarias dentro de dicho espacio.

Los diseños por competencias responden, en términos generales, a diferentes motivaciones que Cano (2008: 2-3) sintetiza en tres:

1) Desarrollar la capacidad de incorporar conocimiento permanente. Aprender y desaprender.

2) Movilizar y combinar los diferentes conocimientos, desarrollando el pensamiento complejo.

3) Alcanzar una formación integral, incorporando conjuntos de conocimientos, habilidades y actitudes de diferente carácter.

En definitiva, diseñan una enseñanza-aprendizaje que permita la adaptación constante de la persona a nuevas situaciones y a los rápidos cambios de la sociedad del conocimiento del s. XXI. Es por ello que podría hablarse de un modelo o paradigma que quiere centrarse en la gestión del conocimiento y cómo esta se realiza durante el aprendizaje y la puesta en práctica del mismo.

La estrategia de la Universitat Politècnica de València (UPV), recogida en su Plan Estratégico UPV2020, asume como primer reto fundamental convertirse en un referente en calidad formativa siempre orientada a las necesidades de la sociedad de nuestro tiempo. Es por ello que este primer reto tiene como objetivo "avanzar hacia modelos de formación que logren que sus estudiantes adquieran las competencias necesarias para poder tener una adecuada inserción laboral" (Plan Estratégico UPV2020, 2015:7). Así, la UPV, incorpora a la evaluación competencias de tipo transversal en sus planes de estudio, en cuanto habilidades que se deben adquirir y que no responden a un ámbito temático o disciplinario específico, sino que están presentes, cruzando, en todos los dominios de las actividades de enseñanza aprendizaje. Tal y como señala en su Proyecto de Competencias Transversales (2015), estas "pretenden sintetizar un perfil competencial para el alumnado egresado de la UPV, garantizando que se cubre el marco de referencia de todas las titulaciones", así como ser transferibles a una variedad de contextos académicos y laborales a lo largo de la vida, pero siendo también competencias personales y sociales, por lo que incluyen habilidades cognitivas y metacognitivas — relacionadas con la capacidad de aprender a aprender, de conocer y regular los propios procesos de 
aprendizaje-, así como también conocimientos instrumentales, metodológicos y actitudinales ${ }^{1}$, necesarios, estos últimos, para la constante adaptación a las sociedades del conocimiento actuales, como se ha señalado en líneas anteriores.

La UPV establece y define un total de trece competencias transversales caracterizadas por favorecer la formación integral — son integradoras —, por ser transferibles a diferentes contextos —de lo académico y profesional a lo personal y social—, interdependientes y multifuncionales, características estas importantísimas puesto que el trabajo sobre una competencia tendrá reflejo y repercusión directa sobre las demás. Además, son evaluables para poder acreditar el nivel alcanzado por el alumnado.

Para desarrollar y evaluar la adquisición de las mismas, se han establecido tres niveles de dominio: el primer nivel correspondería a las asignaturas impartidas en primer y segundo curso de Grado, el segundo nivel a asignaturas del resto de cursos hasta completar el graduado. Y el tercer nivel se desarrolla en Máster.

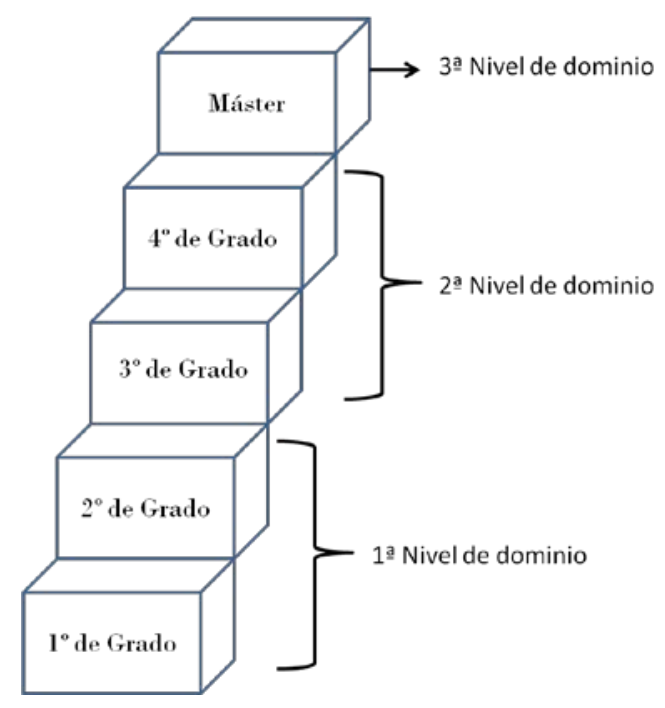

Fig. 1 Niveles de dominio por curso

Con todo lo señalado hasta el momento, esta comunicación presenta y describe el proceso de enseñanza-aprendizaje llevado a cabo para medir una de estas competencias transversales, específicamente al la número siete, situada en el campo de la ética tanto medioambiental como profesional, dentro de la titulación del Grado en Gestión y Administración Pública (GGAP), que se imparte en la Facultad de Administración y Dirección de Empresas (FADE) de la UPV. El contenido que se presenta es fruto de las actividades que se vienen desarrollando en las asignaturas que son punto de control de dicha competencia en el Grado señalado.

${ }^{1}$ UPV. Proyecto de Competencias Transversales, 2015: 3-5. 


\section{Responsabilidad ética, medioambiental y profesional. Competencia CT07}

Actuar con responsabilidad ética, medioambiental y profesional (CT07) ante uno mismo y los demás, implica responsabilidad y destreza a la hora de, por un lado, reducir los efectos negativos producidos por las distintas acciones y actividades que realizamos $\mathrm{y}$, por otro lado, potenciar los beneficios que una actitud responsable, en cumplimiento de las normas, puede generar en la actividad profesional dentro de ámbito de la gestión y administración pública, bajo principios de sostenibilidad en su amplio espectro: social, económico y ambiental. Ser responsable y consecuente con las acciones e inacciones y lo que genera hacia los demás es vital en el desarrollo y evaluación de esta competencia. Así, la UPV señala que la responsabilidad profesional "surge incluida dentro de la responsabilidad moral, alcanzando el interior de nuestra conciencia y con dos objetivos primordiales: evitar toda falta voluntaria y disminuir, en lo posible, el número de faltas involuntarias por debilidad humana, flaqueza propia o negligencia ajena", pero también por negligencia propia. Importantísima esta dimensión en profesionales que van a centrar su trabajo en la administración y gestión de lo público, lo que implica transparencia, rendición de cuentas y sostenibilidad, por lo que la ética debe ser un principio rector incuestionable en el aprendizaje de los estudiantes.

Bajo esta concepción, los niveles de dominio establecidos para los resultados de aprendizaje serán diferentes en cada nivel, de modo que el primero está basado en el cuestionamiento y reconocimiento de otras miradas y perspectivas, mientras que en el segundo será importante el análisis crítico de juicios propios y ajenos, para en el tercer nivel pasar a la construcción argumentativa desde las perspectivas, normas y valores estudiados en el nivel de grado.

Estos tres niveles se concretan en una serie de indicadores que recogemos en la siguiente tabla y a través de los cuales se transluce el carácter que fundamenta el aprendizaje en principios democráticos y de gobernanza, de diálogo permanente y proyección de escenarios.

Tabla 1. Resultados de aprendizaje por niveles de dominio e indicadores en la CT07

\begin{tabular}{|c|c|c|}
\hline & $\begin{array}{l}\text { Resultados de } \\
\text { aprendizaje }\end{array}$ & Indicadores \\
\hline Nivel 1. & $\begin{array}{l}\text { Cuestionar la realidad y ser } \\
\text { conscientes de los } \\
\text { conceptos y valores a partir } \\
\text { de los que se construye la } \\
\text { misma }\end{array}$ & $\begin{array}{l}\text { - Tomar conciencia de otra manera de ver y percibir las cosas. } \\
\text { - Aceptar críticamente nuevas perspectivas, aunque cuestionen las } \\
\text { propias. } \\
\text { - Diferenciar hechos y opiniones o interpretaciones en las } \\
\text { argumentaciones de los demás. } \\
\text { - Reflexionar sobre las consecuencias y efectos que las decisiones y } \\
\text { propuestas tienen sobre las personas. } \\
\text { - Reconocer los conceptos éticos y deontológicos de la profesión }\end{array}$ \\
\hline
\end{tabular}


Analizar críticamente los juicios propios y ajenos

Nivel 2. sobre la realidad, y ser conscientes de las consecuencias e implicaciones de estos
- Interpretar y valorar críticamente la información y la realidad (capacidad crítica).

- Fundamentar y argumentar los juicios propios.

- Reconocer las limitaciones propias y considerar los juicios de los demás (capacidad de autocrítica).

- Incorporar y valorar críticamente los conceptos éticos y deontológicos de la profesión.
Mostrar y argumentar la pertinencia de los comportamientos y juicios que se emiten,

Nivel 3. fundamentados con conceptos éticos y deontológicos.
- Actuar de modo coherente y responsable en sus decisiones y conductas.

- Gestionar de forma adecuada situaciones que desde un punto de vista ético resulten significativas, complejas o conflictivas.

- Satisfacer, mediante el diálogo, alguna necesidad vinculada a la convivencia a partir de los valores éticos deseados.

- Aplicar los conceptos éticos y deontológicos de la profesión.

- No discriminar a las personas por razones de diferencia social, cultural o de género.

Fuente: elaborado a partir del Proyecto Competencias Transversales de la UPV.

A lo largo de los meses de abril y mayo del año 2019, el profesorado responsable de las asignaturas designadas como punto de control de la competencia: responsabilidad ética, medioambiental y profesional, han organizado sesiones para la puesta en común del proceso de medición desarrollado, centrado en la descripción de las metodologías y los sistemas de evaluación llevados a cabo. Las asignaturas implicadas se reparten entre los cuatro cursos del ciclo formativo de Grado en Gestión y Administración (GGAP) de la Facultad de Administración y Dirección de Empresas (FADE). En total han sido cuatro, entre asignaturas obligatorias y optativas, que se resumen en la siguiente tabla.

Tabla 2. Asignaturas punto de control CT07 y nivel de dominio

\section{NIVELES DE DOMINIO}

\begin{tabular}{llll}
\hline & NIVEL-1 & & NIVEL-2 \\
\hline $1^{\circ}$ Curso & Introducción a la Contabilidad & $3^{\circ}$ Curso & Gestión de Calidad \\
\hline $1^{\circ}$ Curso & Sociología & $4^{\circ}$ Curso & $\begin{array}{l}\text { Control de Costes en los Sistemas de Salud } \\
\text { y Servicios Sociales Públicos }\end{array}$ \\
\hline
\end{tabular}

Fuente: elaboración propia.

La sesión de trabajo se dividió en dos partes. La primera destinada a recoger, para cada asignatura de las señaladas en la tabla anterior, tanto la metodología utilizada para la enseñanza aprendizaje, como el tipo de evaluación que se realiza a la hora de emitir valoración cualitativa en relación al alcance que el alumnado ha obtenido en la consecución de la competencia transversal objeto de esta comunicación. La segunda parte, estuvo destinada a elaborar un listado de ventajas e inconvenientes, que se han detectado a lo largo del curso académico, encontrados en la metodología empleada y la evaluación, de modo que permita elaborar una matriz de las potencialidades y 
limitaciones que presentan las actividades que se han llevado a cabo, así como las circunstancias académicas que impulsan mejoras, desde la interacción profesoradoalumnado y viceversa, o por el contrario las frenan.

Las asignaturas señaladas han centrado su metodología y evaluación de la competencia de la siguiente forma que se detalla a continuación, en relación al nivel de dominio donde cada una se inscribe:

— En el Nivel 1 de dominio, dentro del primer curso encontramos dos asignaturas:

- Introducción a la Contabilidad. Centra sus objetivos en la formulación de cuentas anuales aptadas a los formatos legalmente establecidos, realizando supuestos reales de operaciones de contabilización, es decir, utiliza la metodología de estudio de caso. Realiza la evaluación a través de pruebas escritas, cuyos resultados se vuelcan hacia la obtención de la competencia transversal.

- Sociología. Plantea una metodología basada en problemas y $\operatorname{casos}^{2}$ que ha de resolver el alumnado en prácticas de aula y seminarios, a través de la recogida y análisis de fuentes secundarias de información en dos líneas: bibliografía y citación bibliográfica de modo que no se violen los derechos de propiedad intelectual ni se cometa plagio y la explotación de bases de datos oficiales que le permitan un análisis de la realidad actual de la población de nuestras sociedades, así como tener en cuenta los retos que plantea la Agendas 2030 y los objetivos de Desarrollo Sostenible. Estos principios y formas de trabajar, que se inscriben, por un lado, dentro de la metodología de aprender haciendo ${ }^{3} \mathrm{y}$, por otro lado, en el aprendizaje por ensayo y error ${ }^{4}$, se consideran fundamentales a la hora incorporar la responsabilidad ética en la formación del alumnado. A partir del trabajo académico realizado y la calificación cuantitativa obtenida, esta se vuelca a una escala de valoración cualitativa de la siguiente forma: $D=0,00-5,50 ; C=5,60-6,99$; $\mathrm{B}=7,00-8,99 ; \mathrm{A}=9,00-10,00^{5}$.

- En el Nivel 2 de dominio, propio de las asignaturas de tercer y cuarto curso, se recogen otras dos asignaturas:

- Gestión de calidad. Asignatura de tercer curso. Utiliza tres tipos de metodología de enseñanza aprendizaje: a) Aprendizaje servicio ${ }^{6}$-abrir la Universidad a la

\footnotetext{
${ }^{2}$ El estudio de casos ya se utilizaba dentro de la Sociología por investigadores como Herbert Spencer (1851) y Max Weber (1922).

${ }^{3}$ La metodología de aprendizaje "aprender haciendo" tiene su origen en el enfoque constructivista en pedagogía, aunque ya se cita en Aristóteles. Requiere dinámica y "mayor actividad por parte del aprendiz: experiencia directa, simulaciones, dramatizaciones y demostraciones". Rodríguez y Ramírez (2014: 56). En dicho enfoque "los estudiantes son el eje y los protagonistas del proceso, y son quienes deciden cuándo y cómo aprender, mientras que el profesor es sólo un guía que orienta, motiva y retroalimenta a los estudiantes" (Caro y Reyes, 2003: 49)

${ }^{4}$ Donde el estudiante sustituye el aprendizaje de fórmulas por el planteamiento de problemas, lo que le permite extraer interrelaciones y sinergias entre variables de análisis e intervención, de forma que cultive el pensamiento divergente, crítico y la creatividad (Sosa, A. 2001).

${ }^{5} \mathrm{D}=$ no se ha alcanzado el nivel mínimo; $\mathrm{C}=$ en proceso de adquisición, alcanzado parcialmente; $\mathrm{B}=$ nivel adecuado, se alcanza satisfactoriamente; $\mathrm{A}=$ se supera con creces, de modo excelente.

${ }^{6}$ Recogiendo aquí las ideas de Aramburuzabala, Cerrillo y Tello (2015: 85), este tipo de aprendizaje tiene un "carácter experiencial que integra el servicio a la comunidad y la reflexión crítica con el aprendizaje académico, el crecimiento personal y la responsabilidad cívica".
} 
sociedad - donde el trabajo con datos y temáticas de actualidad y su correcto manejo ético es fundamental a la hora de presentar un estudio que aporte servicio a instituciones y administraciones públicas. Aprendizaje que está inscrito y tiene como eje la Agenda 2030 y los Objetivos de Desarrollo Sostenible, así como sus metas; b) Metodología basada en conferencias y ponencias de expertos en sus clases, dando claridad y responsabilidad profesionalidad ética y laboral al trabajo real que se realiza; c) Trabajo de campo basado en información recogida a través de la visitas a organizaciones tanto públicas como privadas. Posteriormente a esta aplicación metodológica, se evalúa la competencia transversal a través de trabajos académicos presentados por el alumnado y cuestionarios concretos para dicha evaluación, además de una matriz o rúbrica, donde se describen criterios y niveles de calidad que completan la evaluación de la competenciat transversal.

- Control de costes en los sistemas de salud y servicios sociales públicos. Asignatura que se ubica en cuarto y último curso del GGAP. Su metodología está basada en el estudio de casos, por lo que también enfrenta una metodología basada en aprender haciendo, así como en el aprendizaje reflexivo ${ }^{7}$ a través de dilemas éticos, considerados, ambos, métodos fundamentales a la hora de la toma de decisiones bajo principios de responsabilidad ética y profesional en el manejo de costes en sistemas públicos de salud y servicios sociales. La evaluación de la competencia transversal, en consecuencia, se realiza a través del resultado del estudio de caso, con defensa oral y observación de la realización de la tarea.

La segunda parte de la puesta en común del profesorado, que tuvo como objetivo general elaborar y listar aquellos aspectos positivos que potencian el trabajo sobre la medición de la competencia transversal, así como sus limitaciones, han arrojado los siguientes resultados consensuados a lo largo de la dinámica realizada:

- Las potencialidades aparecidas pueden ser ordenadas en tres ámbitos o dimensiones:

- Metodológica: las actividades desarrolladas, que pueden afrontar la medición de la competencia transversal, deben estar inscritas dentro de las metodologías de aprendizaje aprender haciendo, aprendizaje servicio y aprendizaje reflexivo.

- Diseño de actividades. El trabajo de caso y de campo, recogiendo y explotando datos reales, se considera fundamental, además ha de ser trabajo secuencial y de grupo.

- Resultados. La aplicación de dichas metodologías y diseños permite poder medir la responsabilidad, la conciencia y la empatía, es decir, cómo afecta el propio comportamiento a la evaluación de los compañeros y a la propia. Por tanto, tomar responsabilidad sobre la decisión elegida y ser consciente y consecuente con los efectos colectivos y por extensión sociales, lo que permite trabajar para que las nuevas generaciones de profesionales reduzcan los casos de mal uso en el

\footnotetext{
${ }^{7}$ En el que se aplican técnicas de debate de modo que el alumnado pueda llegar a la resolución del problema bajo principios de responsabilidad ética profesional, es decir, puede ayudarle a encontrar el camino por la vía de una construcción propia de la solución con base en un diálogo colectivo.
} 
procedimiento de manejo de datos y normativas, y apoyar la creación de cultura de lo público, lo comunitario y la conciencia crítica, siempre en términos de sostenibilidad y su consecución.

— Las limitaciones encontradas se organizan en las siguientes dimensiones:

- Tamaño de grupo: En grupos mayores a 25 alumnos y alumnas juntos en el aula se dificulta la medición y la evaluación de la competencia transversal, derivado del control y nivel de observación que requiere. Así mismo, también, la aplicación de rúbricas para evaluación.

- Profesorado. El manejo de grupos mayores de 25 personas es complicado con un solo profesor o profesora en el aula, a lo que se une la falta de reconocimiento del trabajo docente para la medición de estas competencias transversales que añade actividades concretas para dicho fin.

- Alumnado: posee comportamientos limitantes de base, cuya ruptura en la conciencia colectiva es complicado, requiriendo un esfuerzo pedagógico adicional. Estos son los siguientes:

- Trabajo académico y en grupo. Entienden el grupo como un entorno sin responsabilidad, se diluye la ética cuanto mayor es el grupo.

- No saben gestionar la libertad académica, aspecto este que emerge a la hora de proponer que elijan y decidan una temática para su trabajo académico.

- Infantilización excesiva, lo que diluye la toma de conciencia sobre su responsabilidad académica.

- La desmotivación detectada, como factor que añade efectos limitantes al comportamiento responsable y ético.

- Piensan que copiar y pegar de internet no tiene importancia, lo han normalizado perdiendo comprensión en relación al plagio como delito académico.

- El uso de las tecnologías de la información y comunicación (TIC) - las plataformas webs, redes sociales, WhatsApp, etc.- de forma indiscriminada, constituye un distractor para el aprendizaje haciéndose un mal uso de las mismas, alejado de funciones académicas, de documentación y aprendizaje. El libre acceso constante, en el aula, no permite el control ético de la actividad. No toman responsabilidad sobre el uso de la tecnología y la información web.

- Enseñanza-aprendizaje. Dentro del marco ético, enseñar criterios de de normalización y citación bibliográfica, aprender a no plagiar y concienciar sobre ello no se trabaja desde primero, salvo excepciones. 
Tabla 3. Potencialidades y limitaciones en la evaluación de la CT07

\begin{tabular}{|c|c|c|c|}
\hline \multirow[b]{2}{*}{ Dimensiones } & POTENCIALIDADES & LIMITACIONES & \multirow[b]{2}{*}{ Dimensiones } \\
\hline & & & \\
\hline Metodológica & $\begin{array}{l}\text { - Aprender haciendo } \\
\text { - Aprendizaje servicio } \\
\text { - Aprendizaje reflexivo }\end{array}$ & $>25$ estudiantes & $\begin{array}{l}\text { Tamaño de } \\
\text { grupo }\end{array}$ \\
\hline Actividades & $\begin{array}{l}\text { - Estudio de caso } \\
\text { - Trabajo de campo } \\
\text { - Explotación Bases de datos }\end{array}$ & $\begin{array}{l}\text { - Un solo profesor/a en grupos }>25 \\
\text { estudiantes } \\
\text { - Sin reconocimiento docente por } \\
\text { trabajo en CT }\end{array}$ & Profesorado \\
\hline \multirow[t]{2}{*}{ Resultados } & $\begin{array}{l}\text { - Medición de responsabilidad } \\
\text { - Concienciación, empatía, cultura } \\
\text { de lo público y comunitario } \\
\text { - Conciencia crítica } \\
\text { - Aplicación principios de } \\
\text { sostenibilidad }\end{array}$ & $\begin{array}{l}\text { - El grupo es un espacio sin } \\
\text { responsabilidad } \\
\text { - Incapacidad de gestión de la } \\
\text { libertad académica } \\
\text { - Infantilización e irresponsabilidad } \\
\text { - Desmotivación } \\
\text { - Desconocimiento del plagio } \\
\text { - Inadecuado uso de TICs }\end{array}$ & Alumnado \\
\hline & & $\begin{array}{l}\text { Conciencia antiplagio y } \\
\text { normalización bibliográfica tardía }\end{array}$ & $\begin{array}{l}\text { Enseñanza y } \\
\text { aprendizaje }\end{array}$ \\
\hline
\end{tabular}

Fuente: elaboración propia.

Adicionalmente, se recogió otro tipo de observaciones y consideraciones que se entiende han de tenerse en cuenta a la hora de evaluar la adquisición de esta competencia transversal, debido al marco ético profesional y medioambiental en el que se suscribe. Son las siguientes:

- El uso del espacio y equipamiento común, así como el comportamiento respetuoso con la política medioambiental de la UPV en cuanto al depósito de los residuos para su reciclaje en los contenedores adecuados, por parte de los estudiantes, debe ser un elemento a evaluar dentro de esta competencia.

- Se debe tener en cuenta el uso de la tecnología digital que hace el alumnado en el aula.

- La co-evaluación es una herramienta que puede ayudar a mejorar la evaluación de la misma, en todas sus dimensiones.

- Se aconseja, a su vez, organizar charlas cortas en el aula protagonizadas por el Área de Medio Ambiente.

- Es recomendable trabajar con un proyecto transversal entre asignaturas para medir no solo la competencia transversal que aquí tratamos específicamente, sino todas las demás. 
- Aplicar más a menudo el servicio Turnitin $^{8}$, para la detección de plagio en los trabajos académicos desde primer curso.

\section{Conclusiones}

Tras la dinámica grupal realizada por el profesorado responsable de las asignaturas que son punto de control para la CT07, la percepción y experiencia expuesta colectivamente, establece consenso en relación a la importancia y utilidad de los trabajos prácticos, de grupo y secuenciales a la hora de medir la responsabilidad, la conciencia y la empatía de los alumnos y las alumnas en la realización de las actividades, por lo que han sobresalido tres tipos de metodologías inscritas dentro del aprendizaje activo: aprender haciendo, aprendizaje servicio y aprendizaje reflexivo.

En general, se ha detectado la dificultad de evaluar las competencias transversales en su conjunto, y más concretamente la competencia responsabilidad ética, medioambiental y profesional. Una de las causas radica, al menos parcialmente, en las dudas y reflexiones del profesorado en relación a qué es lo que hay que evaluar y cómo hacerlo, así como a la diversidad de variables que han de recogerse. Ello a pesar de la existencia de herramientas en PoliformaT y un sitio específico en la web de la Universitat con información sobre cada una de las competencias — videos explicativos, documento sobre estrategias, etc., pero que, finalmente, algunos resultan ser más conceptuales que metodológicos - entre otros recursos disponibles.

Junto a esta dificultad, otra de las observadas por el colectivo de profesores participantes se deriva del volumen de alumnos existente en algunas de las asignaturas, especialmente evidente en las asignaturas obligatorias del grado. Este hecho dificulta el trabajo y la evaluación de ésta y otras competencias transversales, convirtiéndose la rúbrica en un modelo disfuncional derivado de dicho volumen. Cuando se trabaja con grupos de alumnos más reducidos (de hasta 25 personas), se incrementa significativamente la posibilidad de diseñar actividades más enfocadas y más adecuadas para la evaluación de competencias de este tipo y posibilita que dicha evaluación se realice de una manera mucho más objetiva y también más justa, así como de la aplicación directa de rúbricas para la misma. El tamaño del grupo se percibe como un hándicap a la hora de aplicar el sistema de rúbricas en la evaluación, por lo que es una herramienta que ha sido escasamente utilizada en las asignaturas señaladas.

Una de las herramientas, a la que todos los profesores tienen acceso y que permite valorar la originalidad de los trabajos realizados e identificar la existencia de plagios, es el servicio Turnitin, recomendándose su uso con más asiduidad, puesto que permite el control y evaluación de la ética que el estudiante aplica en la demostración de su

\footnotetext{
${ }^{8}$ Servicio de detección de plagio. Identifica contenido poco original a través de la detección de similitudes, lo que permite administrar el riesgo de la mala conducta académica comparando el contenido producido por el alumnado con la única base de datos que incluye Internet, trabajos estudiantiles y artículos científicos indexados $<$ https://www.turnitin.com/es $>$ [Consulta: 30 de mayo de 2019].
} 
aprendizaje, por lo que en algunas de las asignaturas se hace especial énfasis en la importancia de este aspecto.

A pesar de las desventajas detectadas, el equipo de docentes que ha coordinado esta comunicación trabaja en potenciar sus ventajas, ante la necesidad en las sociedades actuales de impulsar la conciencia y la acción profesional futura de sus estudiantes, dentro de parámetros éticos, sociales, políticos, económicos y medioambientales como vía para alcanzar espacios más sostenibles.

\section{Referencias}

ARAMBURUZABALA, P., CERRILLO, R. y TELLO I. (2015). “Aprendizaje-servicio: una propuesta metodológica para la introducción de la sostenibilidad curricular en la Universidad". Profesorado. Revista de currículum y formación de profesorado, vol. 19 (1), p. 78-95 < https://www.ugr.es/ recfpro/rev191ART5.pdf $>$ [Consulta: 30 de mayo de 2019].

CANO GARCÍA, Ma . E. (2008). "La evaluación por competencias en la educación superior" en PROFESORADO. Revista de Currículum y Formación de Profesorado, vol. 12 (3), p. 1-16 <https://www.ugr.es/ recfpro/rev123COL1.pdf $>$ [Consulta: 02 de mayo de 2019].

CARO SPINEL, S. Y REYES ORTIZ, J.C. (2003). "Prácticas docentes que promueven el aprendizaje activo en ingeniería civil". Revista de Ingeniería, número 18, p. 48-55 <file://C:/Users/Usuario/Downloads/479-1438-1-SM.pdf> [Consulta: 30 de mayo de 2019].

GIMENO SACRISTÁN, J., PÉREZ GÓMEZ, A. I., MARTÍNEZ RODRÍGUEZ, J. B., TORRES SANTOMÉ, J., ANGULO RASCO, F., ÁlVAREZ MÉNDEZ, J.M. (2008). Educar por competencias, ¿qué hay de nuevo? Madrid: Editorial Morata.

PORTILLO-POBLADOR, N., MARTÍN-MARÍN, J., ALONSO CAMINERO, J.M. (2017) "Educación para el desarrollo sostenible de las futuras generaciones desde las aulas universitarias. Una experiencia en colaboración con Cruz Roja". Conferencia internacional RED-U 2017. Bilbao. 13-14 de noviembre 2017.

RODRÍGUEZ GARCÍA, A. B. y RAMÍREZ LÓPEZ, L. J. (2014). “Aprender haciendoInvestigar reflexionando: Caso de estudio paralelo en Colombia y Chile". Revista $\begin{array}{llllllll}\text { Academia } & y & \text { Virtualidad, } & \text { vol. } & 7 & \text { (2), } & \text { p. } & 53-63\end{array}$ file://C:/Users/Usuario/Downloads/Dialnet-

AprenderHaciendoInvestigarReflexionando-5061041\%20(1).pdf $>$ [Consulta: 30 de mayo de 2019].

SOSA ESPINOSA, A. (2001) "Sociología como optativa de primer ciclo, en la E.T.S.I. Caminos, Canales y Puertos. Una experiencia docente”. I Jornadas de Innovación educativa en la Universidad Politécnica de Valencia. Libro de Resúmenes. UPV-VICAA 
UPV. (2015). Plan Estratégico UPV 2015-2020. Universitat Politècnica de València $<$ https://www.upv.es/organizacion/la-

institucion/documentos/Plan_Estrategico_UPV2020 int.pdf $>$ [Consulta: 20 de mayo de 2019].

UPV. (2015). Proyecto Competencias transversales. Universitat Politècnica de València

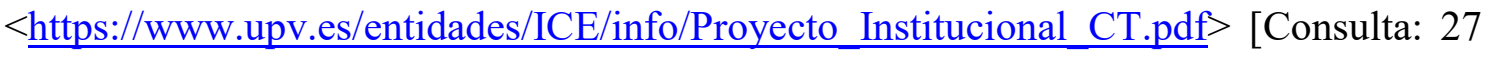
de mayo de 2019]. 